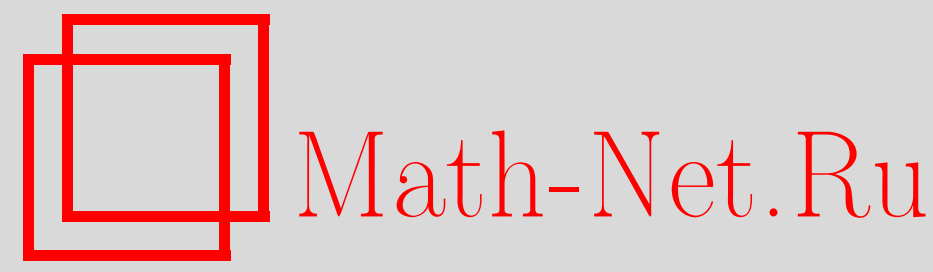

В. В. Горяйнов, Функция Кёнигса и дробное итерирование вероятностных производящих функций, Матем. сб., 2002, том 193, номер 7, 69-86

DOI: https://doi.org/10.4213/sm667

Использование Общероссийского математического портала Math-Net.Ru подразумевает, что вы прочитали и согласны с пользовательским соглашением

http://www.mathnet.ru/rus/agreement

Параметры загрузки:

IP: 54.92 .164 .108

26 апреля 2023 г., $12: 34: 53$ 
УДК $517.54+519.21$

\author{
В.В. Горяйнов
}

\title{
Функция Кёнигса и дробное итерирование вероятностных производящих функций
}

\begin{abstract}
Функция Кёнигса возникает как предел нормированной определенньг образом последовательности итераций голоморфной функции. Она также является решением соответствующего функционального уравнения и может служить для получения итераций порождающей ее функции.

В работе дано описание класса функций Кёнигса, отвечающих вероятностнтм производящим функциям, допускающим вложение в однопараметрическую полугруппу дробных итераций. Полученные результаты можно рассматривать как критерий вложимости процесса Гальтона-Ватсона в однородный марковский ветвящийся процесс.

Библиограф̆ия: 20 названий.
\end{abstract}

\section{Введение}

Итерации отображения можно рассматривать лишш в случае согласованности областей определения и значений. В контексте аналитических функций изучаются следующие три ситуации. Локальный случай, когда областью определения является окрестность неподвижной точки (для каждой итерации своя). Случай мероморфных функций, когда областью определения является вся комплексная плоскость $\mathbb{C}$. Наконец, случай, когда функция аналитична в некоторой области и принимает значения из этой же области. В последнем случае в качестве области определения обычно выбирается круг или полуплоскость. В настоящей работе изучаются функции, аналитические в единичном круге $\mathbb{D}=\{z \in \mathbb{C}:|z|<1\}$. Более точно, основным предметом изучения являются вероятностные производящие функции, т.е. те, которые имеют тейлоровское разложение вида $f(z)=\sum_{k=0}^{\infty} p_{k} z^{k}$, где $p_{k} \geqslant 0$ и $\sum_{k=0}^{\infty} p_{k}=1$. Если $p_{0} \neq 1$, т.е. соответствующее распределение вероятностей невырожденное и $f(z) \not \equiv 1$, то ограничения на коэффициенты $p_{k}$ влекут аналитичность $f$ в единичном круге и условие $f(\mathbb{D}) \subset \mathbb{D}$.

Через $f^{n}$ будем обозначать $n$-кратную итерацию функции $f$, т.е. $f^{0}(z) \equiv z$, $f^{1}=f$ и $f^{n}=f \circ f^{n-1}$ при $n=2,3, \ldots$ Изучение асимптотического поведения итераций при $n \rightarrow \infty$ приводит (см., например, [1]) к так назьваемой функиии Кёнигса. Возникая как предел нормированной тем или иньм способом последовательности итераций $f^{n}, n=1,2, \ldots$, она является решением определенного функционального уравнения с известной функцией $f$. Важным обстоятельством при этом является то, что все итерации $f^{n}, n=2,3, \ldots$, можно получить как решения функционального уравнения с той же функцией Кёнигса.

Целью данной работы является описание множества функций Кёнигса, соответствующих вероятностным производящим функциям, которые допускают дробное

(C) В. В. ГОряйнов 2002 
итерирование в классе вероятностных производящих функций. Другими словами, решается задача вложения функции в однопараметрическую полугруппу вероятностных производящих функций в терминах ее функции Кёнигса. Поскольку каждую вероятностную производящую функцию можно ассоциировать с процессом Гальтона-Ватсона, описьвающего развитие популяции однотипных частиц, то решаемая задача вложения эквивалентна получению условий вложимости соответствующего процесса в однородный марковский ветвящийся процесс с непрерывњым временем. Исследование этого вопроса имеет достаточно длительную историю (см. [2], [3; гл. 5], [4], [5], [6, гл. 3]). В работах [7], [8] были получены критерии вложимости в терминах асимптотического поведения натуральных итераций производящей функции и в терминах решений некоторого функционального уравнения, определяемого производящей функцией и ее производной. Функция Кёнигса также определяет процесс Гальтона-Ватсона посредством соответствующего функционального уравнения. Это направление в теории ветвящихся процессов получило развитие в ряде работ [9], [10]. С этой точки зрения полученные в данной работе результаты можно рассматривать как описание вложимых процессов Гальтона-Ватсона в терминах функциональных уравнений.

Общая проблема дробных итераций аналитической функции, естественно, имеет еще более длительную историю. Ее решение существенно зависит от того, в каком классе функций она рассматривается. Первые результаты относились к локальной постановке и были получены в работах [11], [12] в конще 19-го века. Оказалось, что за редким исключением (если производная в неподвижной точке не обращается в нуль и не равна по модулю единице) функция допускает вложение в однопараметрическую полугруппу дробных итераций. Результат для мероморфных функций [13] оказался диаметрально противоположньм - вложение допускают только дробно-линейные преобразования. Случай единичного круга качественно отличается и по результатам и по методам. Его активное исследование началось значительно позже (см., например, [14] и приведенную там библиографию). Здесь в отличие от других двух случаев возникают трудности, связанные с граничньм поведением аналитических функций. При изучении итераций важную роль играет природа неподвижных точек отображения. В случае аналитической в единичном круге функции может не оказаться внутренней неподвижной точки. Тогда ее заменяет некоторая выделенная точка $q$ на границе $\partial \mathbb{D}$ единичного круга, в которой сушествуют угловые пределы самой функции и ее производной и, кроме того, $f(q)=q, 0<f^{\prime}(q) \leqslant 1$. Вид решения поставленной выше задачи будет зависеть от того, существует ли внутренняя неподвижная точка у вероятностной производящей функции или нет, а в случае ее отсутствия, является ли выделенная граничная точка притягивающей $\left(f^{\prime}(q)<1\right)$ или нет $\left(f^{\prime}(q)=1\right)$.

В $\S 1$ вводится терминология, уточняется постановка задачи и формулируются основные результаты работы. Во $§ 2$ рассматривается случай, когда вероятностная производящая функция имеет внутреннюю неподвижную точку. В $\S 3$ изучается случай граничной притягивающей точки. Случай $f^{\prime}(q)=1$, известный в теории ветвящихся процессов как критический, рассматривается в $\S 4$. В $\S 5$ полученные результаты иллюстрируются на некоторых примерах, в которых удается найти явный вид функции Кёнигса и разрешить функциональное уравнение для дробных итераций. 
Автор выражает искреннюю признательность А.А. Гончару и Е. М. Чирке за предоставленную возможность обсуждения результатов работы на семинаре по теории функций в МИАН, а также А. А. Гончару за полезные советы по улучшению структуры статьи.

\section{§1. Терминология и формулировка основных результатов}

Совокупность всех голоморфных в единичном круге $\mathbb{D}$ функций, принимающих значения из $\mathbb{D}$, будем обозначать через $\mathfrak{P}$. Заметим, что $\mathfrak{P}$ образует топологическую полугруппу относительно операции композиции и топологии локально равномерной в $\mathbb{D}$ сходимости. Совокупность всех вероятностных производящих функций, исключая тождественно постоянную $f(z) \equiv 1$, образует подполугруппу $\mathfrak{P}^{+}$ в полугрупше $\mathfrak{P}$. Роль единищы в этой некоммутативной полугруппе играет тождественное преобразование $f(z) \equiv z$. Рассматривая $\mathbb{R}^{+}=\{t \in \mathbb{R}: t \geqslant 0\}$ как топологическую полугруппу относительно операции сложения и обычной топологии вешественных чисел, под однопараметрической полугруппой в $\mathfrak{P}$ (или $\mathfrak{P}^{+}$) будем понимать непрерывный гомоморфизм $t \mapsto f^{t}$, действующий из $\mathbb{R}^{+}$в $\mathfrak{P}$ (или $\mathfrak{P}^{+}$). Будем говорить также, что $f$ как элемент полугруппы $\mathfrak{P}$ (или $\mathfrak{P}^{+}$) безгранично делим в соответствующей полугруппе, если для каждого натурального $n$ найдется такой элемент $g_{n}$ полугруппы $\mathfrak{P}$ (соответственно, $\mathfrak{P}^{+}$), что

$$
f=g_{n} \circ \cdots \circ g_{n}=g_{n}^{n}
$$

Оказывается, что безграничная делимость в полугруппе $\mathfrak{P}^{+}$эквивалентна (см. [8], $[15])$ вложимости $f$ в однопараметрическую полугруппу в $\mathfrak{P}^{+}$. Другими словами, $f$ является безгранично делимой в $\mathfrak{P}^{+}$тогда и только тогда, когда найдется такая однопараметрическая полугруппа $t \mapsto f^{t}$ в $\mathfrak{P}^{+}$, что $f^{1}=f$. Поскольку семейство $\left\{f^{t}\right\}_{t \geqslant 0}$ обладает свойством $f^{t+s}=f^{t} \circ f^{s}$ для всех $s, t \geqslant 0$, то $f^{t}, t \geqslant 0$, называют дробными итерациями функиии $f$. В случае существования такой однопараметрической полугруппы будем говорить, что $f$ вложима в непрерьвную nолугруппу итераций в $\mathfrak{P}^{+}$или, просто, вложима.

Важньм результатом при изучении динамики голоморфного отображения круга $\mathbb{D}$ в себя является теорема Данжуа-Вольфа ([16], [17], см. также [1]), согласно которой, если $f \in \mathfrak{P}$ отлична от мёбиусова преобразования единичного круга на себя, то последовательность ее итераций $f^{n}, n=1,2, \ldots$, сходится локально равномерно в $\mathbb{D}$ к тождественно постоянной функции, т.е. $f^{n}(z) \rightarrow q$ при $n \rightarrow \infty$. В случае, когда $q$ принадлежит $\mathbb{D}$, она является единственной неподвижной внутренней точкой функции $f$. В случае же, когда $|q|=1$, в этой точке существуют угловые пределы

$$
f(q)=\lim _{z \rightarrow q} f(z), \quad f^{\prime}(q)=\lim _{z \rightarrow q} f^{\prime}(z)
$$

При этом $f(q)=q$ и $0<f^{\prime}(q) \leqslant 1$. В литературе $q$ называют точкой Данжууа-Вольфа функции $f$. Заметим также, что все итерации функции $f$ (включая и дробные, если они сушествуют) имеют одну и ту же точку Данжуа-Вольфа. В связи с этим естественно выделить в $\mathfrak{P}$ подполугрупшы $\mathfrak{P}[q],|q| \leqslant 1$, функций с одной и той же точкой Данжуа-Вольфа $q$. Если $f \in \mathfrak{P}^{+}$, то $f^{n}(0)$ принадлежат отрезку $[0,1]$ при всех натуральных $n$. Поэтому точка Данжуа-Вольфа вероятностной 
производящей функции принадлежит отрезку $[0,1]$ и

$$
\mathfrak{P}^{+}=\bigcup_{0 \leqslant q \leqslant 1} \mathfrak{P}^{+}[q]
$$

где $\mathfrak{P}^{+}[q]=\mathfrak{P}^{+} \cap \mathfrak{P}[q]$.

Пусть $f \in \mathfrak{P}^{+}[q], 0 \leqslant q<1$, и $f^{\prime}(q)=\gamma>0$. Тогда (см., например, [1; 44$]$ ) существует предел

$$
\lim _{n \rightarrow \infty} \frac{f^{n}(z)-q}{\gamma^{n}}=K(z),
$$

которьй представляет собой непостоянную аналитическую в $\mathbb{D}$ функцию, удовлетворяющую функциональному уравнению Шрёдера

$$
K(f(z))=\gamma K(z)
$$

При этом $K$ называют функиией Кёнигса функиии $f$ и она является единственньп решением уравнения (2) в классе аналитических в единичном круге функций с $K(q)=0, K^{\prime}(q)=1$. Итерации функции $f$ удовлетворяют уравнению

$$
K\left(f^{n}(z)\right)=\gamma^{n} K(z)
$$

В случае $f^{\prime}(q)=0$ функция $f$ не является вложимой. Для формулировки критерия вложимости $f$ в терминах ее функции Кёнигса введем в рассмотрение следуюшие классы функций.

Определим вначале семейство функций

$$
\varphi_{n}(z, q)=\frac{z^{n}+\sum_{k=0}^{n-1} q^{n-k} z^{k}}{1+\sum_{k=1}^{n} q^{k}}
$$

где $q \in[0,1]$ и $n=1,2, \ldots$ Через $\mathfrak{C}[q], 0 \leqslant q \leqslant 1$, будем обозначать класс функций $\varphi$, допускающих представление в виде $\varphi(z)=\sum_{n=1}^{\infty} \lambda_{n} \varphi_{n}(z, q)$, в котором $\lambda_{n} \geqslant 0$ и $\sum_{n=1}^{\infty} \lambda_{n} \leqslant 1$.

Tеорема 1. Пусть $f \in \mathfrak{P}^{+}[q], 0 \leqslant q<1, u f^{\prime}(q)=\gamma>0$. Тогда $f$ допускает вложение в однопараметрическую полугруппу в $\mathfrak{P}^{+}$в том и только том случае, если ее функиия Кёнигса (1) допускает представление в виде

$$
K(z)=(z-q) \exp \left(\int_{q}^{z} \frac{\varphi(\zeta)-\varphi(q)}{(\zeta-q)(1-\varphi(\zeta))} d \zeta\right)
$$

с некоторой $\varphi$ из $\mathfrak{C}[q]$.

Доказательство этой теоремы приводится в следуюшем параграфе. Показано также, что функция $K$, имеющая представление (3), является однолистной и отображает $\mathbb{D}$ на звездную относительно начала координат область, а дробные итерации функции $f$ определяются посредством $K$ формулой $f^{t}(z)=K^{-1}\left(\gamma^{t} K(z)\right)$, $t \geqslant 0$.

В случае $q=1$ функция $f$ имеет в точке $z=1$ угловую производную $f^{\prime}(1)=\mu$, $0<\mu \leqslant 1$. Если в левой части (1) вместо $q$ подставить 1 , а вместо $\gamma$ подставить $\mu$, 
то для функции $f$ из $\mathfrak{P}^{+}[1]$ предел, по-прежнему, будет существовать. Однако предельная функция $K$ может оказаться тождественно постоянной. Поэтому определение функции Кёнигса для $f \in \mathfrak{P}^{+}[1]$ нуждается в корректировке.

Допустим, что $f \in \mathfrak{P}^{+}[1]$ и $f^{\prime}(1)=\mu<1$. Тогда сушествует предел

$$
\lim _{n \rightarrow \infty} \frac{f^{n}(z)-1}{f^{n}(0)-1}=Q(z)
$$

которьй представляет собой непостоянную аналитическую в $\mathbb{D}$ функцию и удовлетворяет функциональному уравнению

$$
Q(f(z))=\mu Q(z)
$$

Функция $Q$, очевидно, удовлетворяет следуюшим условиям: $Q(0)=1, \operatorname{Re} Q(z)>0$ при $z \in \mathbb{D}$ и все ее производные неположительны на интервале $(0,1)$. В классе таких функций уравнение (5) также имеет единственное решение (см., напримep, [9], [10]).

Tеорема 2. Пусть $f \in \mathfrak{P}^{+}[1] u f^{\prime}(1)=\mu<1$. Tогда $f$ допускает вложсение в однопараметрическую полугруппу в $\mathfrak{P}^{+}$в том и только том случае, если найдется $\varphi$ из $\mathfrak{C}[1]$ с $\varphi(1)<1$ и такая, что для функиии Кёнигса (4) выполняется равенство

$$
Q(z)=\exp \left(\int_{0}^{z} \frac{1-\varphi(1)}{(\zeta-1)(1-\varphi(\zeta))} d \zeta\right)
$$

В третьем параграфе доказывается эта теорема и устанавливается, что дробные итерации функции $f$, как и в предыдущем случае, определяются однозначно посредством $Q$ равенством $f^{t}(z)=Q^{-1}\left(\mu^{t} Q(z)\right)$.

В случае $f \in \mathfrak{P}^{+}[1]$ и $f^{\prime}(1)=1$ предел в (4) снова может оказаться тождественно постоянной функцией. Этот случай известен в теории ветвящихся процессов как критический $\left(f^{\prime}(1)<1\right.$ соответствует докритическому случаю). С точки зрения анализа его можно рассматривать как случай двойной неподвижной точки, поскольку равенство $f^{\prime}(1)=1$ означает кратность корня $z=1$ уравнения $f(z)-z=0$. Его изучению посвящены работы [18], [19].

Tеорема 3. Пусть $f \in \mathfrak{P}^{+}[1], f^{\prime}(1)=1 u f^{\prime}(0) \neq 0$. Тогда существует предел

$$
\lim _{n \rightarrow \infty} \frac{f^{n}(z)-f^{n}(0)}{\left(f^{n}\right)^{\prime}(0)}=H(z),
$$

который представляет собой непостоянную аналитическую в $\mathbb{D}$ функцию, удовлетворяющую функииональному уравнению

$$
H(f(z))=H(z)+H(f(0)) .
$$

Кроме того, уравнение (8) имеет единственное решение в классе аналитических в $\mathbb{D}$ функиий $H$ с тейлоровским разложсением вида

$$
H(z)=\sum_{n=1}^{\infty} a_{n} z^{n}, \quad a_{1}=1, \quad a_{n} \geqslant 0, \quad n=2,3, \ldots
$$


Функцию $H$, определяемую равенством (7) в критическом случае, мы также будем называть функиией Кёнигса вероятностной производящей функиии $f$. Итерации $f^{n}, n=2,3, \ldots$, будут удовлетворять уравнению

$$
H\left(f^{n}(z)\right)=H(z)+n H(f(0)) .
$$

В случае существования дробных итераций в этом равенстве $n$ можно заменить на $t, t \geqslant 0$.

Tеорема 4. Пусть $f \in \mathfrak{P}^{+}[1], f^{\prime}(1)=1 u f^{\prime}(0)>0$. Тогда $f$ допускает вложсение в однопараметрическую полугруппу в $\mathfrak{P}^{+}$в том и только том случае, если найдется $\varphi$ из $\mathfrak{C}[1]$ с $\varphi(1)=1$ и такая, что для функиии Кёнигса (7) выполняется равенство

$$
H(z)=\int_{0}^{z} \frac{1-\varphi(0)}{(1-\zeta)(1-\varphi(\zeta))} d \zeta
$$

\section{§2. Случай внутренней точки Данжуа-Вольфа}

Основной целью данного параграфа является доказательство теоремы 1. Будем предполагать, что вероятностная производящая функция $f(z) \not \equiv z$ принадлежит $\mathfrak{P}^{+}[q]$ и $0 \leqslant q<1$.

Допустим вначале, что $f$ вложима и $K$ - ее функция Кёнигса, определенная равенством (1). Заметим, что условие вложимости влечет однолистность (см., например, [7]) функции $f$ и, следовательно, $f^{\prime}(z) \neq 0$ для всех $z \in \mathbb{D}$ (в частности, $\left.f^{\prime}(q)=\gamma \neq 0\right)$. Свойство однолистности распространяется и на итерации $f^{n}$, $n=2,3, \ldots$, функции $f$, а поскольку $K(z) \not \equiv$ const, то и на функцию Кёнигса $K$. Кроме того, по теореме Вейерштрасса о локально равномерной сходимости последовательности аналитических функций имеем

$$
K^{\prime}(z)=\lim _{n \rightarrow \infty} \frac{\left(f^{n}\right)^{\prime}(z)}{\gamma^{n}}
$$

Учитывая, что $\left(f^{n}\right)^{\prime}(z) \neq 0$ и $K^{\prime}(z) \neq 0$ при $z \in \mathbb{D}$, последнее соотношение можно переписать в виде

$$
\lim _{n \rightarrow \infty} \frac{\gamma^{n}}{\left(f^{n}\right)^{\prime}(z)}=\frac{1}{K^{\prime}(z)}
$$

Но тогда

$$
\frac{K(z)}{K^{\prime}(z)}=\lim _{n \rightarrow \infty} \frac{f^{n}(z)-q}{\left(f^{n}\right)^{\prime}(z)}
$$

Допустим теперь, что $t \mapsto f^{t}$ - однопараметрическая полугруппа в $\mathfrak{P}^{+}$, для которой $f^{1}=f$. По теореме 4 из [7] инфинитезимальная образующая

$$
v(z)=\left.\frac{\partial}{\partial t} f^{t}(z)\right|_{t=0}
$$


этой однопараметрической полугруппы имеет вид

$$
v(z)=\alpha\left(q-z+(1-q) \sum_{k=2}^{\infty} \lambda_{k} \frac{z^{k}-q^{k}}{1-q^{k}}\right),
$$

где $\alpha>0, \lambda_{k} \geqslant 0$ и $\sum_{k=2}^{\infty} \lambda_{k}=1$. При доказательстве теоремы 6 в [7] было показано также, что выполняется следующее предельное соотношение

$$
\lim _{t \rightarrow \infty} \frac{f^{t}(z)-q}{\left(f^{t}\right)^{\prime}(z)}=\frac{v(z)}{v^{\prime}(q)}
$$

Сравнивая его с полученным вьшше, приходим к равенству

$$
\frac{K(z)}{K^{\prime}(z)}=\frac{v(z)}{v^{\prime}(q)}
$$

связьвающему функцию Кёнигса и инфинитезимальную образуюшую.

Преобразуем теперь вид инфиннитезимальной образующей

$$
\begin{aligned}
v(z) & =\alpha(z-q)\left(\sum_{k=2}^{\infty} \lambda_{k} \frac{z^{k-1}+q z^{k-2}+\cdots+q^{k-1}}{1+q+\cdots+q^{k-1}}-1\right) \\
& =\alpha(z-q)\left(\sum_{k=1}^{\infty} \lambda_{k+1} \varphi_{k}(z, q)-1\right)
\end{aligned}
$$

где $\varphi_{k}(z, q), k=1,2, \ldots$, определены как и в $\S 1$. Замечая что,

$$
\varphi(z)=\sum_{k=1}^{\infty} \lambda_{k+1} \varphi_{k}(z, q)
$$

принадлежит $\mathfrak{C}[q]$, приходим к представлению

$$
v(z)=\alpha(z-q)(\varphi(z)-1)
$$

Отсюда следует, что $v^{\prime}(q)=\alpha(\varphi(q)-1)$ и, следовательно,

$$
\frac{v(z)}{v^{\prime}(q)}=\frac{(z-q)(\varphi(z)-1)}{\varphi(q)-1}=(z-q) \frac{1-\varphi(z)}{1-\varphi(q)}
$$

Теперь полученное вьше соотношение между функцией Кёнигса и инфинитезимальной образующей можно переписать в виде

$$
\frac{(z-q) K^{\prime}(z)}{K(z)}=\frac{1-\varphi(q)}{1-\varphi(z)} .
$$

Отсюда, в частности, следует, что $K(z) /(z-q)$ является аналитической и не обращается в нуль в круге $\mathbb{D}$. Поэтому в $\mathbb{D}$ выделяется однозначная ветвь логарифма 
$\ln (K(z) /(z-q))$, которая обращается в нуль при $z=q$. При этом, ее производная имеет вид

$$
\left(\ln \frac{K(z)}{z-q}\right)^{\prime}=\frac{\varphi(z)-\varphi(q)}{(z-q)(1-\varphi(z))} .
$$

Интегрируя это соотношение, получаем

$$
\ln \frac{K(z)}{z-q}=\int_{q}^{z} \frac{\varphi(\zeta)-\varphi(q)}{(\zeta-q)(1-\varphi(\zeta))} d \zeta
$$

что после потенцирования дает

$$
K(z)=(z-q) \exp \left(\int_{q}^{z} \frac{\varphi(\zeta)-\varphi(q)}{(\zeta-q)(1-\varphi(\zeta))} d \zeta\right)
$$

Таким образом, в одну сторону теорема 1 доказана.

Обратно, пусть функция Кёнигса, определяемая формулой (1), имеет интегральное представление $(3)$ с некоторой функцией $\varphi$ из $\mathfrak{C}[q]$. Покажем, что $K$ однолистна в $\mathbb{D}$ и переводит единичный круг в звездную относительно начала координат область. Пусть $\zeta=L(z)=(z-q) /(1-q z)$ и $G(\zeta)=K \circ L^{-1}(\zeta)$. Тогда доказываемое свойство функции $K$ эквивалентно тому, что $G$ - звездная функция или (см., например, [20]) для всех $\zeta \in \mathbb{D}$ вьполняется неравенство $\operatorname{Re}\left(\left(\zeta G^{\prime}(\zeta)\right) /(G(\zeta))\right)>0$. Поскольку

$$
G^{\prime}(\zeta)=\frac{K^{\prime}(z)}{L^{\prime}(z)}=\frac{(1-q z)^{2}}{1-q^{2}} K^{\prime}(z),
$$

то условие звездности функции $G$ переписывается в виде

$$
\operatorname{Re}\left((z-q)(1-q z) \frac{K^{\prime}(z)}{K(z)}\right)>0
$$

для всех $z \in \mathbb{D}$. Учитывая представление функции $K$ равенством $(3)$, последнее соотношение принимает вид $\operatorname{Re}((1-q z) /(1-\varphi(z)))>0$, что, в свою очередь, эквивалентно условию

$$
\operatorname{Re}\left(\frac{1-\varphi(z)}{1-q z}\right)>0
$$

при $z \in \mathbb{D}$. Проверку этого условия достаточно провести лишњ для функций $\varphi_{n}(z, q), n=1,2, \ldots$, поскольку $\varphi$ представляет собой выпуклую комбинацию последних. После очевидных преобразований

$$
1-\varphi_{n}(z, q)=\frac{1+q+\cdots+q^{n-1}-z\left(z^{n-1}+q z^{n-2}+\cdots+q^{n-1}\right)}{1+q+\cdots+q^{n}}
$$

доказываемое неравенство перепишется в виде

$$
\operatorname{Re}\left(\frac{1+q+\cdots+q^{n-1}-z\left(z^{n-1}+q z^{n-2}+\cdots+q^{n-1}\right)}{1-q z}\right)>0 .
$$

В силу принципа минимума для гармонических функций достаточно показать, что

$$
\operatorname{Re}\left((1-q \bar{\varkappa})\left(1+q+\cdots+q^{n-1}-\varkappa\left(\varkappa^{n-1}+q \varkappa^{n-2}+\cdots+q^{n-1}\right)\right)\right) \geqslant 0
$$


для всех $\varkappa \in \partial \mathbb{D}$, т.е. $|\varkappa|=1$. Однако

$$
\begin{aligned}
& (1-q \bar{\varkappa})\left(1+q+\cdots+q^{n-1}-\varkappa\left(\varkappa^{n-1}+q \varkappa^{n-2}+\cdots+q^{n-1}\right)\right) \\
& =\left(1-\varkappa^{n}\right)+(1-\bar{\varkappa})\left(q+q^{2}+\cdots+q^{n}\right) .
\end{aligned}
$$

Поскольку $\operatorname{Re}\left(1-\varkappa^{n}\right) \geqslant 0, \operatorname{Re}(1-\bar{\varkappa}) \geqslant 0$, то требуемое неравенство доказано.

Таким образом, функция $K$ однолистна в единичном круге и отображает его на звездную относительно начала координат область. Это позволяет определить для всех $t \geqslant 0$ функции $f^{t}(z)=K^{-1}\left(e^{-\sigma t} K(z)\right)$, где $\sigma=-\ln \gamma$. Легко видеть, что $t \mapsto f^{t}$ является однопараметрической полугруппой в $\mathfrak{P}$, а ее инфинитезимальная образуюшая $v$ выражается через $K$ по формуле

$$
v(z)=\left.\frac{\partial}{\partial t} f^{t}(z)\right|_{t=0}=-\sigma \frac{K(z)}{K^{\prime}(z)} .
$$

Из интегрального представления функции $K$ следует, что

$$
v(z)=\frac{\sigma}{1-\varphi(q)}(z-q)(\varphi(z)-1) .
$$

Но тогда (см. [7]) $t \mapsto f^{t}$ является однопараметрической полугруппой в $\overline{\mathfrak{P}^{+}[q]}$, замькании полугруппы $\mathfrak{P}^{+}[q]$. Далее, по предположению $K$ является функцией Кёнигса для $f$ и потому выполняется равенство (2). Из однолистности функции $K$ и определения функций $f^{t}, t \geqslant 0$, получаем $f(z)=K^{-1}(\gamma K(z))=f^{1}(z)$. Поскольку $f \in \mathfrak{P}^{+}[q]$, то $t \mapsto f^{t}$ является однопараметрической полугруппой в $\mathfrak{P}^{+}[q]$ и вложимость $f$ доказана.

В заключение этого параграфа сделаем некоторые замечания относительно интегрального представления (3). Если $\varphi-$ произвольная функция из $\mathfrak{C}[q], 0 \leqslant q<1$, то соответствующая ей функция $K$ является однолистной в единичном круге и отображает его на звездную относительно начала координат область. Для любого $\alpha>0$ определены функции $f^{t}=K^{-1}\left(e^{-\alpha t} K(z)\right), t \geqslant 0$. При этом $t \mapsto f^{t}$ будет однопараметрической полугруппой в $\overline{\mathfrak{P}^{+}[q]}$. Таким образом, формула (3) дает точное описание класса функций Кёнигса, отвечающих вложимым функциям в $\overline{\mathfrak{P}^{+}[q]}$. Другими словами, каждая функция $K$ вида (3) определяет единственный с точностью до замены временной шкалы однородный марковский ветвящийся процесс с непрерывным временем и вероятностью вырождения $q$.

\section{§3. Докритический случай}

В этом параграфе мы докажем теорему 2, т.е. рассмотрим случай, когда точка Данжуа-Вольфа является граничной и строго притягивающей. Таким образом, на протяжении этого параграфа мы будем считать, что $f \in \mathfrak{P}^{+}[1]$ и $f^{\prime}(1)=\mu<1$. Кроме того, будем предполагать, что в разложении $f(z)=\sum_{k=0}^{\infty} p_{k} z^{k}$ коэффицииенты $p_{0}, p_{1}$ удовлетворяют неравенству $p_{0}+p_{1}<1$, поскольку случай $p_{0}+p_{1}=1$, т.е. $f(z)=p_{0}+p_{1} z$, подробно рассмотрен в последнем параграфе.

Допустим вначале, что $f$ вложима и $Q$ - ее функция Кёнигса, определяемая равенством (4). Поскольку для всех $n=1,2, \ldots$ в единичном круге вьполняется неравенство

$$
\operatorname{Re}\left(\frac{f^{n}(z)-1}{f^{n}(0)-1}\right)>0
$$


то и $\operatorname{Re} Q(z)>0$ при $z \in \mathbb{D}$. В силу теоремы Вейерштрасса

$$
Q^{\prime}(z)=\lim _{n \rightarrow \infty} \frac{\left(f^{n}\right)^{\prime}(z)}{f^{n}(0)-1}
$$

и, следовательно,

$$
\frac{Q^{\prime}(z)}{Q(z)}=\lim _{n \rightarrow \infty} \frac{\left(f^{n}\right)^{\prime}(z)}{f^{n}(z)-1} .
$$

Пусть $t \mapsto f^{t}$ - однопараметрическая полугруппа в $\mathfrak{P}^{+}$, для которой $f^{1}=f$. Как следует из [7], ее инфинитезимальная образуюшая $v$ допускает представление в виде

$$
v(z)=\alpha\left(1-z+\sum_{k=2}^{\infty} \frac{\lambda_{k}}{k}\left(z^{k}-1\right)\right),
$$

где $\alpha>0, \lambda_{k} \geqslant 0$ и $\sum_{k=2}^{\infty} \lambda_{k}<1$. Кроме того, выполняется равенство

$$
\lim _{t \rightarrow \infty} \frac{\left(f^{t}\right)^{\prime}(z)}{f^{t}(z)-1}=\frac{v^{\prime}(1)}{v(z)}
$$

Таким образом, мы снова приходим к соотношению

$$
\frac{Q^{\prime}(z)}{Q(z)}=\frac{v^{\prime}(1)}{v(z)}
$$

которое связывает функцию Кёнигса и инфинитезимальную образуюшую. Как и в предыдушем случае, инфинитезимальную образуюшую можно преобразовать к виду $v(z)=\alpha(z-1)(\varphi(z)-1)$, где $\varphi \in \mathfrak{C}[1]$ и $\varphi(1)=\sum_{k=2}^{\infty} \lambda_{k}<1$. Замечая также, что

$$
v^{\prime}(1)=\lim _{x \rightarrow 1} \frac{v(x)}{x-1}=\alpha(\varphi(1)-1),
$$

полученное выше соотношение между $Q$ и $v$ перепишем в виде

$$
\frac{Q^{\prime}(z)}{Q(z)}=\frac{1-\varphi(1)}{(z-1)(1-\varphi(z))}
$$

Интегрирование этого соотношения с учетом условия $Q(0)=1$ приводит к интегральному представлению (6) функции Кёнигса.

Обратно, пусть функция Кёнигса $Q$, определяемая итерациями функции $f$ по формуле (4), допускает представление (6) с некоторой функцией

$$
\varphi(z)=\sum_{k=1}^{\infty} \lambda_{k} \varphi_{k}(z, 1)=\frac{1}{z-1} \sum_{k=1}^{\infty} \frac{\lambda_{k}}{k+1}\left(z^{k+1}-1\right),
$$

где $\lambda_{k} \geqslant 0$ и $\sum_{k=1}^{\infty} \lambda_{k}=\varphi(1)<1$. Как следует из [7], функция

$$
v(z)=(z-1)(\varphi(z)-1)=1-z+\sum_{k=1}^{\infty} \frac{\lambda_{k}}{k+1}\left(z^{k+1}-1\right)
$$


является инфинитезимальной образующей некоторой однопараметрической полугрупшы $t \mapsto g^{t}$ в $\mathfrak{P}^{+}[1]$. Дифференцируя обе части эволюционного уравнения

$$
\frac{\partial}{\partial t} g^{t}(z)=v\left(g^{t}(z)\right)
$$

по $z$, приходим к дифференциальному уравнению

$$
\frac{\partial}{\partial t}\left(g^{t}\right)^{\prime}(z)=v^{\prime}\left(g^{t}(z)\right)\left(g^{t}\right)^{\prime}(z)
$$

Отсюда с учетом условия $\left.\left(g^{t}\right)^{\prime}(z)\right|_{t=0} \equiv 1$ получаем

$$
\ln \left(\left(g^{t}\right)^{\prime}(z)\right)=\int_{0}^{t} v^{\prime}\left(g^{s}(z)\right) d s
$$

где под логарифмом понимается непрерывная ветвь, обращающаяся в нуль при $t=0$. Далее, поскольку для всех $s>0$ выполняются соотношения $g^{s}(x) \nearrow 1$ и $v^{\prime}\left(g^{s}(x)\right) \nearrow v^{\prime}(1)$ при $x \nearrow 1$, то в последнем равенстве можно осушествить предельный переход под знаком интеграла при $z=x \quad$ \, что приводит к равенствам

$$
\left(g^{t}\right)^{\prime}(1)=\mathrm{e}^{t v^{\prime}(1)}=\mathrm{e}^{t(\varphi(1)-1)}
$$

Функция $g=g^{1}$ принадлежит $\mathfrak{P}^{+}[1]$ и $g^{\prime}(1)=\mathrm{e}^{v^{\prime}(1)}=\nu<1$. По доказанному ее функция Кёнигса однозначно определяется инфинитезимальной образующей $v$ и, следовательно, совпадает с $Q$, т.е. та же, что и у функции $f$. Кроме того, для каждого $\tau>0$ функция $g^{\tau}$ является вложимой в однопараметрическую полугруппу $t \mapsto g^{t \tau}$, инфинитезимальная образуюшая которой лиш множителем отличается от $v$. Следовательно, для $g^{\tau}$ функцией Кёнигса также является $Q$ и выполняется равенство $Q\left(g^{\tau}(z)\right)=\nu^{\tau} Q(z)$. В частности, при $\tau=\ln \mu / \ln \nu=\tau_{0}$ получаем $Q\left(g^{\tau_{0}}(z)\right)=\mu Q(z)$. Если теперь мы докажем однолистность функции $Q$, то отсюда и из уравнения (5) будет следовать, что $f(z)=Q^{-1}(\mu Q(z))=g^{\tau_{0}}(z)$. Тем самым вложимость $f$ будет доказана.

Для доказательства однолистности $Q$ заметим прежде всего, что условия $\operatorname{Re} Q(z)>0$ и $Q(0)=1$ позволяют выделить в $\mathbb{D}$ однозначную ветвь $\ln Q(z)$, обращающуюся в нуль при $z=0$. Далее, поскольку $L(z)=\ln (1-z)$ - выпуклая функция и

$$
\frac{(\ln Q(z))^{\prime}}{L^{\prime}(z)}=\frac{1-\varphi(1)}{1-\varphi(z)}
$$

имеет положительную вешественную часть в $\mathbb{D}$, то $\ln Q(z)$ является почти выпуклой (см., например, [20]) и, следовательно, однолистной функцией. Отсюда следует однолистность $Q$ и теорема 2 доказана.

Завершим параграф некоторыми замечаниями относительно интегрального представления (6). Пусть $\varphi$ - произвольная из $\mathfrak{C}[1]$ с $\varphi(1)<1$ и $Q$ - функция, определяемая посредством $\varphi$ формулой (6). Как было показано выше, $v(z)=$ $(z-1)(\varphi(z)-1)$ является инфинитезимальной образующей однопараметрической полугрупы $t \mapsto g^{t}$ в $\mathfrak{P}^{+}[1]$ и каждая $g^{t}$ имеет в качестве функции Кёнигса $Q$. Если же $f$ из $\mathfrak{P}^{+}[1]$ с $f^{\prime}(1)<1$ имеет в качестве функции Кёнигса также $Q$, то она должна 
совпадать с одной из функций семейства $\left\{g^{t}\right\}_{t>0}$. Таким образом, формула (6) описывает в точности класс функций Кёнигса, отвечающих вложимым функциям из $\mathfrak{P}^{+}[1]$, для которых $z=1$ является строго притягиваюшей точкой. Кроме однолистности $Q$ обладает еше геометрическим свойством, которое заключается в звездности области $Q(\mathbb{D})$ относительно начала координат, являющимся граничной точкой этой области. Действительно, если $w_{0}=Q\left(z_{0}\right), z_{0} \in \mathbb{D}$, то кривая $\Gamma: t \mapsto g^{t}\left(z_{0}\right), 0 \leqslant t<\infty$, отображается посредством $Q$ в отрезок, соединяюший $w_{0}$ с началом координат, поскольку при всех $t \geqslant 0$ вьполняется $Q\left(g^{t}\left(z_{0}\right)\right)=\mathrm{e}^{t v^{\prime}(1)} w_{0}$.

\section{§4. Критический случай}

ДокаЗАТЕЛЬСТво ТЕОРемЫ 3 . Пусть $f(z)=\sum_{k=0}^{\infty} p_{k} z^{k} \not \equiv z$ принадлежит $\mathfrak{P}^{+}[1]$ и $f^{\prime}(1)=1$. Поскольку $p_{k} \geqslant 0, k=0,1, \ldots$, то сама функция, ее итерации и все производные неотрицательны и не убывают на промежутке $(0,1)$. Кроме того, для любого $x \in[0,1)$ последовательность $\left\{f^{n}(x)\right\}$ строго монотонно возрастает и ее пределом является 1 , т.е. точка Данжуа-Вольфа. Если $f^{\prime}(0)=0$, то $f$ не будет вложимой. Поэтому в дальнейшем предполагаем, что $f^{\prime}(0) \neq 0$. Но тогда и для всех ее итераций будет выполняться условие $\left(f^{n}\right)^{\prime}(0) \neq 0$. Следовательно, для всех $n=1,2, \ldots$ определены функции

$$
h_{n}(z)=\frac{f^{n}(z)-f^{n}(0)}{\left(f^{n}\right)^{\prime}(0)}
$$

которые аналитичны в $\mathbb{D}$ и имеют тейлоровское разложение в окрестности $z=0 \mathrm{c}$ неотрицательньми коэффициентами.

Покажем, что последовательность $\left\{h_{n}\right\}$ сходится локально равномерно в $\mathbb{D}$. Для доказательства этого заметим вначале, что для всех $x \in(0,1)$ и $n=1,2, \ldots$ выполняется

$$
\frac{h_{n+1}(x)}{h_{n}(x)}=\frac{\left(f^{n+1}(x)-f^{n+1}(0)\right)\left(f^{n}\right)^{\prime}(0)}{\left(f^{n+1}\right)^{\prime}(0)\left(f^{n}(x)-f^{n}(0)\right)}=\frac{f\left(f^{n}(x)\right)-f\left(f^{n}(0)\right)}{f^{\prime}\left(f^{n}(0)\right)\left(f^{n}(x)-f^{n}(0)\right)}>1 .
$$

Последнее неравенство следует из выпуклости $f$ на интервале $(0,1)$ и показывает, что для любого $x \in(0,1)$ последовательность $\left\{h_{n}(x)\right\}$ монотонно возрастает. В силу теоремы Витали локально равномерная сходимость в $\mathbb{D}$ последовательности $\left\{h_{n}\right\}$ будет следовать из ее локально равномерной ограниченности.

Фиксируем произвольно $r \in(0,1)$. Из неотрицательности тейлоровских коэффициентов функции $h_{n}, n=1,2, \ldots$, следует

$$
\max _{|z| \leqslant r}\left|h_{n}(z)\right|=h_{n}(r)
$$

Выберем теперь номер $k>1$ так, чтобы выполнялось неравенство $r<f^{k}(0)$. Это можно сделать, поскольку $f^{n}(0) \nearrow 1$ при $n \rightarrow \infty$. Тогда для любого $n=1,2, \ldots$ будем иметь

$$
\begin{aligned}
h_{n}(r) & \leqslant h_{n}\left(f^{k}(0)\right)=\frac{f^{n}\left(f^{k}(0)\right)-f^{n}(0)}{\left(f^{n}\right)^{\prime}(0)}<\frac{\left(f^{n}\right)^{\prime}\left(f^{k}(0)\right) f^{k}(0)}{\left(f^{n}\right)^{\prime}(0)} \\
& =\frac{\left(f^{n+k}\right)^{\prime}(0) f^{k}(0)}{\left(f^{n}\right)^{\prime}(0)\left(f^{k}\right)^{\prime}(0)}=\frac{\left(f^{k}\right)^{\prime}\left(f^{n}(0)\right) f^{k}(0)}{\left(f^{k}\right)^{\prime}(0)}<\frac{1}{\left(f^{k}\right)^{\prime}(0)},
\end{aligned}
$$


откуда и следует локально равномерная ограниченность в $\mathbb{D}$ последовательности $\left\{h_{n}\right\}$ и, следовательно, ее сходимость. Предел

$$
H(z)=\lim _{n \rightarrow \infty} h_{n}(z)
$$

является аналитической в $\mathbb{D}$ функцией с неотрицательными тейлоровскими коэффициентами. При этом $H(0)=0$ и $H^{\prime}(0)=1$. Последнее влечет, в частности, что $H(z) \not \equiv$ const. Кроме того, поскольку

$$
\begin{aligned}
H(z) & =\lim _{n \rightarrow \infty} h_{n+1}(z)=\lim _{n \rightarrow \infty} \frac{f^{n+1}(z)-f^{n+1}(0)}{\left(f^{n+1}\right)^{\prime}(0)} \\
& =\lim _{n \rightarrow \infty}\left(\frac{f^{n}(f(z))-f^{n}(0)}{f^{\prime}\left(f^{n}(0)\right)\left(f^{n}\right)^{\prime}(0)}-\frac{f^{n}(f(0))-f^{n}(0)}{f^{\prime}\left(f^{n}(0)\right)\left(f^{n}\right)^{\prime}(0)}\right)=H(f(z))-H(f(0)),
\end{aligned}
$$

то $H$ является решением уравнения (8).

Доказательство единственности решения уравнения (8) в классе аналитических в $\mathbb{D}$ функций с неотрицательными тейлоровскими коэффициентами можно провести по следуюшей схеме (см., например, [6]). Допустим, что $R$ является аналитической в $\mathbb{D}$, имеет неотрицательные тейлоровские коэффициенты, $R(0)=0, R^{\prime}(0)=1$ и вьполняется равенство

$$
R(f(z))=R(z)+R(f(0))
$$

Тогда для всех $n=1,2, \ldots$ будут вьполняться равенства

$$
R\left(f^{n}(z)\right)=R(z)+n R(f(0))
$$

Фиксируем произвольно $x \in(0,1)$ и выберем номер $k$ так, чтобы выполнялись неравенства $f^{k}(0) \leqslant x<f^{k+1}(0)$. Используя монотонность производных $H^{\prime}$ и $R^{\prime}$ на промежутке $(0,1)$, получаем

$$
\frac{R^{\prime}\left(f^{n+k}(0)\right)}{H^{\prime}\left(f^{n+k+1}(0)\right)} \leqslant \frac{R^{\prime}\left(f^{n}(x)\right)}{H^{\prime}\left(f^{n}(x)\right)} \leqslant \frac{R^{\prime}\left(f^{n+k+1}(0)\right)}{H^{\prime}\left(f^{n+k}(0)\right)},
$$

$n=1,2, \ldots$. Дифференцирование функциональных уравнений для итераций дает

$$
H^{\prime}\left(f^{n}(z)\right)\left(f^{n}\right)^{\prime}(z)=H^{\prime}(z), \quad R^{\prime}\left(f^{n}(z)\right)\left(f^{n}\right)^{\prime}(z)=R^{\prime}(z) .
$$

Используя эти соотношения, полученные выше неравенства можно переписать в виде

$$
\frac{\left(f^{n+k+1}\right)^{\prime}(0)}{\left(f^{n+k}\right)^{\prime}(0)} \leqslant \frac{R^{\prime}(x)}{H^{\prime}(x)} \leqslant \frac{\left(f^{n+k}\right)^{\prime}(0)}{\left(f^{n+k+1}\right)^{\prime}(0)} .
$$

Однако

$$
\lim _{n \rightarrow \infty} \frac{\left(f^{n+k+1}\right)^{\prime}(0)}{\left(f^{n+k}\right)^{\prime}(0)}=\lim _{n \rightarrow \infty} f^{\prime}\left(f^{n+k}(0)\right)=1
$$

и, следовательно, $R^{\prime}(x)=H^{\prime}(x)$ для всех $x \in(0,1)$. В силу теоремы единственности для аналитических функций приходим к равенству $R^{\prime}(z)=H^{\prime}(z)$, которое с учетом условия $H(0)=R(0)=0$ приводит к равенству $R(z) \equiv H(z)$. Теорема 3 , таким образом, доказана. 
ДОКАЗАТЕЛЬСТВо ТЕОРЕМЫ 4. Как и выше, мы будем предполагать, что $f(z) \not \equiv z$ принадлежит $\mathfrak{P}^{+}[1], f^{\prime}(1)=1$ и $f^{\prime}(0) \neq 0$. Следовательно, соотношение (7) определяет непостоянную функцию Кёнигса $H$, которая удовлетворяет функциональному уравнению (8).

Допустим вначале, что $f$ вложима, и покажем возможность представления $H$ в виде (9). Снова в силу теоремы Вейерштрасса имеем соотношение

$$
H^{\prime}(z)=\lim _{n \rightarrow \infty} \frac{\left(f^{n}\right)^{\prime}(z)}{\left(f^{n}\right)^{\prime}(0)}
$$

Далее, пусть $t \mapsto f^{t}$ - однопараметрическая полугруппа в $\mathfrak{P}^{+}$, для которой $f^{1}=f$. Тогда, как было показано в [7], ее инфинитезимальная образуюшая имеет вид

$$
v(z)=\alpha\left(1-z+\sum_{k=2}^{\infty} \frac{\lambda_{k}}{k}\left(z^{k}-1\right)\right)
$$

где $\alpha>0, \lambda_{k} \geqslant 0, \sum_{k=2}^{\infty} \lambda_{k}=1$, и выполняется равенство

$$
\lim _{t \rightarrow \infty} \frac{\left(f^{t}\right)^{\prime}(0)}{\left(f^{t}\right)^{\prime}(z)}=\frac{v(z)}{v(0)}
$$

Следовательно, в рассматриваемом случае функция Кёнигса связана с инфинитезимальной образующей соотношением

$$
H^{\prime}(z)=\frac{v(0)}{v(z)}
$$

Как и в предыдущем случае, инфинитезимальную образуюшую можно преобразовать к виду $v(z)=\alpha(z-1)(\varphi(z)-1)$, где $\varphi \in \mathfrak{C}[1]$ и $\varphi(1)=1$. Интегрируя теперь соотношение

$$
H^{\prime}(z)=\frac{1-\varphi(0)}{(1-z)(1-\varphi(z))}
$$

с учетом условия $H(0)=0$, приходим к интегральному представлению (9).

Прежде чем приступить к доказательству достаточности представления (9), заметим, что, как и в предыдущих случаях, все дробные итерации $f^{t}, t>0$, имеют одну и ту же функцию Кёнигса $H$. При этом по доказанному

$$
H\left(f^{t}(z)\right)=H(z)+H\left(f^{t}(0)\right) .
$$

Далее,

$$
\frac{d}{d t} H\left(f^{t}(0)\right)=H^{\prime}\left(f^{t}(0)\right) v\left(f^{t}(0)\right) .
$$

Поскольку

$$
H^{\prime}\left(f^{t}(0)\right)=\frac{v(0)}{v\left(f^{t}(0)\right)},
$$

TO

$$
\frac{d}{d t} H\left(f^{t}(0)\right)=v(0) .
$$


Интегрируя это соотношение, получаем $H\left(f^{t}(0)\right)=t v(0), t \geqslant 0$. Следовательно, все дробные итерации удовлетворяют уравнению

$$
H\left(f^{t}(z)\right)=H(z)+t v(0)
$$

При $t=1$ отсюда, в частности, следует, что $H(f(0))=v(0)$, и мы приходим к функциональному уравнению

$$
H\left(f^{t}(z)\right)=H(z)+t H(f(0))
$$

Допустим теперь, что функция Кёнигса $H$, определяемая итерациями функции $f$ по формуле (7), допускает представление (9) с некоторой функцией $\varphi$ из $\mathfrak{C}[1]$ и $\varphi(1)=1$. Из [7] следует, что тогда

$$
v(z)=(z-1)(\varphi(z)-1)
$$

будет инфинитезимальной образующей некоторой однопараметрической полугруппы $t \mapsto g^{t}$ в $\mathfrak{P}^{+}[1]$ и $\left(g^{t}\right)^{\prime}(1) \equiv 1$. Поскольку $g^{t}(0) \nearrow 1$ при $t \rightarrow \infty$ и $g^{0}(0)=0$, то найдется такое $\tau>0$, что $g^{\tau}(0)=f(0)$. Покажем, что тогда $g^{\tau}(z) \equiv f(z)$, и вложимость $f$ тем самьм будет доказана. Поскольку $f$ и $g^{\tau}$ имеют одну и ту же функцию Кёнигса $H$ ( $f$ по условиям теоремы, а $g^{\tau}$ в силу связи функции Кёнигса и инфинитезимальной образующей), то

$$
H\left(g^{\tau}(z)\right)=H(z)+H\left(g^{\tau}(0)\right)=H(z)+H(f(0))=H(f(z)) .
$$

Равенство $f=g^{\tau}$ будет теперь установлено, если доказать однолистность функции $H$. Замечая, что $L(z)=-\ln (1-z)$ является выпуклой функцией, а в силу представления (9) имеет место равенство

$$
\frac{H^{\prime}(z)}{L^{\prime}(z)}=\frac{1-\varphi(0)}{1-\varphi(z)},
$$

правая часть которого имеет положительную вещественную часть в единичном круге, приходим к выводу о принадлежности функции $H$ классу почти выпуклых функций. Таким образом, однолистность $H$, а вместе с этим и теорема 4 доказаны.

В заключение этого параграфа отметим, что каждая функция $H$, допускающая представление (9), может рассматриваться как функция Кёнигса некоторой вложимой функции $f$ из $\mathfrak{P}^{+}[1]$ с $f^{\prime}(1)=1$. Следовательно, она определяет однородный марковский критический ветвящийся процесс с непрерывным временем.

\section{§5. Некоторые примеры и замечания}

Этот параграф̆ носит иллюстративный характер. Здесь рассмотрены некоторые частные случаи, которые формально выпали из рассмотрения при доказательстве основных результатов. Показано, как эти и другие рассматриваемые ниже примеры вписываются в формулировки теорем.

Начнем рассмотрение с вероятностной производящей функции вида $f(z)=$ $p_{0}+p_{1} z$. Если $p_{0}=1$, то $f(z) \equiv 1$ и не допускает вложения в однопараметрическую 
полугруппу в $\mathfrak{P}^{+}$. При $p_{1}=1$ мы имеем тривиальный случай вложимой функции $f(z) \equiv z$. Поэтому остается рассмотреть случай $0<p_{1}<1$. Поскольку $p_{1}=f^{\prime}(1)<1$, то мы имеем дело с докритическим случаем. Итерации $f^{n}$ и функция Кёнигса $Q$ в этом случае легко находятся

$$
f^{n}(z)=\left(1-p_{1}^{n}\right)+p_{1}^{n} z, \quad Q(z)=1-z .
$$

Из формулы (6) она получается при $\varphi(z) \equiv 0$, т.е. когда в представлении $\varphi$ все числа $\lambda_{k}$ равны нулю. Дробные итерации легко находятся из уравнения Шрёдера:

$$
f^{t}(z)=1-p_{1}^{t}+p_{1}^{t} z .
$$

Важную роль в приложениях играют дробно-линейные вероятностные производящие функции

$$
f(z)=\frac{p}{1-(1-p) z},
$$

$0<p<1$, соответствующие геометрическим распределениям вероятностей. В зависимости от значения $p$ мы имеем все три случая. Рассмотрим вначале критический случай, который соответствует значению $p=1 / 2$, т.е. $f(z)=1 /(2-z)$. Поскольку мёбиусовы преобразования образуют группу, то решение уравнения (8) естественно искать в классе дробно-линейных функций. Замечая, что $z=1$ является неподвижной точкой для $f$ и $H(f(0)) \neq 0$, приходим к следующему выводу: в этой точке $H$ должна иметь полюс. Если дополнить эту информацию равенствами $H(0)=0, H^{\prime}(0)=1$, то мы однозначно приходим к виду $H(z)=z /(1-z)$. Простой проверкой можно убедиться, что эта функция действительно является решением уравнения (8). Из формулы (9) она получается при $\varphi(z)=\varphi_{1}(z, 1)=(z+1) / 2$. Замечая, что $H^{-1}(w)=w /(1+w)$, находим дробные итерации

$$
f^{t}(z)=H^{-1}(H(z)+t H(f(0)))=\frac{t+(1-t) z}{1+t-t z}, \quad t \geqslant 0 .
$$

В случае $0<p<1 / 2$ функция $f$ имеет внутреннюю неподвижную точку $q=$ $p /(1-p)$, которая для нее и является точкой Данжуа-Вольфа. При $1 / 2<p<1$ точкой Данжуа-Вольфа является $q=1$. В обоих случаях функция $f$ имеет еще одну неподвижную точку $r=1$ при $0<p<1 / 2$, или $r=p /(1-p)$ при $1 / 2<p<1$. И в том, и в другом случаях функция Кёнигса $R$ ( $K$ или $Q$ в прежних обозначениях) является решением уравнения Шрёдера

$$
R(f(z))=c R(z)
$$

где

$$
c=f^{\prime}(q)=\left\{\begin{array}{l}
\frac{p}{1-p} \text { при } 0<p<\frac{1}{2} ; \\
\frac{1-p}{p} \text { при } \frac{1}{2}<p<1 .
\end{array}\right.
$$

Поскольку в точке $z=q$ функция $R$ обращается в нуль, то в другой неподвижной точке $z=r$ функции $f$ она должна иметь полюс. Следовательно, $R$ лишь множителем отличается от функции $S(z)=(z-q) /(z-r)$. Замечая, что $S^{-1}(w)=$ 
$(q-r w) /(1-w)$, находим однопараметрическую полугруппу дробных итераций функции $f$ в рассматриваемых случаях

$$
f^{t}(z)=S^{-1}\left(c^{t} S(z)\right)=\frac{p\left((1-p)^{t}-p^{t}\right)+\left((1-p) p^{t}-p(1-p)^{t}\right) z}{(1-p)^{t+1}-p^{t+1}-(1-p)\left((1-p)^{t}-p^{t}\right) z}, \quad t>0 .
$$

К получению дробных итераций и вложимых функций можно подойти с другой стороны, начиная с построения функции Кёнигса. Например, функция $\varphi(z)=z^{n}$, $n=1,2, \ldots$, принадлежит $\mathfrak{C}[0]$ и совпадает с $\varphi_{n}(z, 0)$. Подставляя ее в формулу (3), получаем функцию Кёнигса

$$
K(z)=z \exp \left(\int_{0}^{z} \frac{\zeta^{n}}{\zeta\left(1-\zeta^{n}\right)} d \zeta\right)=\frac{z}{\left(1-z^{n}\right)^{1 / n}}
$$

где под дробной степенњю в знаменателе понимается однозначная ветвь, принимающая значение 1 при $z=0$. Соответствующая функция $f$ и ее дробные итерации находятся из уравнения Шрёдера

$$
f^{t}(z)=\frac{\gamma^{t} z}{\left(1-\left(1-\gamma^{n t}\right) z^{n}\right)^{1 / n}}
$$

где $\gamma=f^{\prime}(0)$. В теории ветвящихся процессов этот случай соответствует обобщенным процессам Юла.

Функция $\varphi(z)=\varphi_{2}(z, 1)=\left(z^{2}+z+1\right) / 3$ относится к критическому случаю, поскольку $\varphi(1)=1$. Она порождает по формуле (9) функцию Кёнигса

$$
H(z)=\frac{2}{3} \frac{z}{1-z}+\frac{2}{9} \ln \frac{2+z}{2(1-z)}
$$

Соответствующая однопараметрическая полугруппа находится из уравнения

$$
H\left(f^{t}(z)\right)=H(z)+t c
$$

где в качестве $c$ можно выбрать произвольное положительное число.

\section{Список литературы}

1. Валирон Ж. Аналитические функции. М.: ГИТ-ТЛ, 1957.

2. Harris T. E. Some mathematical models for branching processes // 2-d Berk. Symp. 1951. P. 305-328.

3. Харрис Т. Теория ветвящихся случайных процессов. М.: Мир, 1966.

4. Karlin S., McGregor J. Embeddability of discrete time simple branching processes into continuous time processes // Trans. Amer. Math. Soc. 1968. V. 132. P. 115-136.

5. Karlin S., McGregor J. Embedding iterates of analytic functions with two fixed points into continuous semigroups // Trans. Amer. Math. Soc. 1968. V. 132. P. 137-145.

6. Athreya K. B., Ney P.E. Branching processes. Berlin: Springer-Verlag, 1972.

7. Горяйнов В. В. Дробное итерирование вероятностных производящих функций и вложение дискретных ветвящихся процессов в непрерывные // Матем. сб. 1993. Т. 184. № 5 . C. $55-74$.

8. Goryainov $V$. $V$. Semigroups of probability generating functions, and infinitely splittable random variables // Theory of Random Processes. 1995. V. 1(17). P. 2-9. 
9. Seneta E. Functional equations and the Galton-Watson processes // Adv. in Appl. Probab. 1969. V. 1. P. 1-42.

10. Kuczma M. Functional equations in a single variable. Warszawa: PWN-Polish Scientific Publishers, 1968.

11. Königs $G$. Recherches sur les intégrales des certaines equations fonctionelles // Ann. Ecole Norm. Sup. (3). 1884. V. 1. P. 3-41.

12. Schröder E. Über itierte Funktionen // Math. Ann. 1871. V. 3. P. 296-322.

13. Baker I. N. Fractional iteration near a fixpoint of multtiplier 1 // J. Austral. Math. Soc. 1964. V. 4. P. 143-148.

14. Cowen C. C. Iteration and the solution of functional equations for functions analytic in the unit disk // Trans. Amer. Math. Soc. 1981. V. 265. P. 69-95.

15. Горяйнов В. В. Однопараметрические полугруппы аналитических функций и композиционный аналог безграничной делимости // Труды ИПММ НАН Украины. 2000. Т. 5. C. $44-57$.

16. Denjoy A. Sur l'iteration des fonctions analytiques // C. R. Acad. Sci. Paris Sér. A. 1926. V. 182. P. 255-257.

17. Wolff J. Sur l'iteration des fonctions // C. R. Acad. Sci. Paris Sér. A. 1926. V. 182. P. 42-43, 200-201.

18. Baker I. N., Pommerenke Ch. On the iteration of analytic functions in a halfplane II // J. London Math. Soc. (2). 1979. V. 20. P. 255-258.

19. Pommerenke Ch. On asymptotic iteration of analytic functions in the disk // Analysis (Munich). 1981. V. 1. P. 45-61.

20. Pommerenke Ch. Univalent functions. Gottingen: Vandenhoeck and Ruprecht, 1975.

Волжский гуманитарншй институт,

Поступила в редакцию

Волгоградская обл.

23.10 .2001

E-mail: vgor@vgumi.vlink.ru 